\title{
A New Experimental Method to Determine the Henry's Law Constant of a Volatile Organic Compound Adsorbed in Soil
}

\author{
S. Ouoba, ${ }^{1}$ F. Cherblanc, ${ }^{2}$ J. Koulidiati, ${ }^{1}$ and J.-C. Bénet ${ }^{2}$ \\ ${ }^{1}$ Laboratoire de Physique et Chimie de l'Environnement, UFR-SEA, Université de Ouagadougou, \\ 03 BP 7021 Ouagadougou, Burkina Faso \\ ${ }^{2}$ Laboratoire de Mécanique et Génie Civil, CNRS, Université Montpellier 2, Place Eugéne Bataillon, 34000 Montpellier, France
}

Correspondence should be addressed to S. Ouoba; samuel_ouoba@univ-ouaga.bf

Received 25 February 2015; Revised 9 April 2015; Accepted 13 April 2015

Academic Editor: José Morillo Aguado

Copyright (C) 2015 S. Ouoba et al. This is an open access article distributed under the Creative Commons Attribution License, which permits unrestricted use, distribution, and reproduction in any medium, provided the original work is properly cited.

\begin{abstract}
This paper presents a new mechanical method to determine Henry's law constant (HLC) of a volatile organic compound (VOC). This method is an extension of the one proposed by Ouoba et al. (2010) to determine the water activity in porous media. This work focuses on TCE and aims at characterizing its liquid-vapor equilibrium in various cases in the form of a pure liquid phase or dissolved in an aqueous solution, adsorbed or not in a natural soil. A liquid phase is disposed in a closed chamber whose volume can be incrementally increased. The recording of the total gas pressure leads to evaluating the vapor partial pressure of a volatile compound even in the case of an aqueous solution. This method has been validated using various aqueous solutions of TCE and the HLC obtained is in agreement with the literature. Then, the validity of Henry's law has been asserted in the case of an aqueous solution of TCE adsorbed in a hygroscopic soil. Indeed, a linear relation between the vapor partial pressure of TCE and its concentration has been obtained while the HLC is about $16 \%$ lower. This result highlights the influence of adsorption phenomena on vapor/liquid equilibrium.
\end{abstract}

\section{Introduction}

Henry's law constant (HLC) represents the equilibrium partition coefficient of a volatile compound between air and water. It reflects the relative volatility of a particular compound and represents a major property to describe fate and transport modeling in environmental risk assessment $[1$, 2]. At equilibrium, Henry's law establishes a sharing relation between the aqueous phase and its gas phase. It is generally described by a linear function and leads to defining the HLC, $K_{H}\left[\mathrm{~Pa} \cdot \mathrm{m}^{3} \cdot \mathrm{mol}^{-1}\right][3]$.

Literature data report that, compared to the large amount of environmentally relevant compounds, only few experimentally determined HLCs are available [4]. While various predictive tools to estimate the HLC have been proposed $[5,6]$, the lack of experimental data clearly calls for additional investigations and associated experimental methods. One of the main reasons for this deficiency of data is the costly and time-consuming investigations needed for reliable measurements. In particular, substances with very low solubilities are difficult to deal with.

So far, a large range of experimental methods has been proposed. Main approaches include static methods like headspace gas chromatography (HSGC) $[7,8]$, the equilibration partitioning in closed systems method (EPICS) [9-11] and further development by combination with solid-phase microextraction [12]. Simultaneously, dynamic equilibration methods have been widely used based on bubble columns [13-16] and generalized later by the inert gas stripping method [17, 18] One of the recent methods proposed by Richon [19] achieves a considerable improvement of HLC measurement in terms of speed, accuracy, and simplicity. Anyway, all these different methods rely on expensive measuring devices including gas chromatography $[1,10,11$, $15,17,18,20-23]$, liquid chromatography [14], or infrared spectrometer [16]. These apparatus require to be regularly calibrated and are not easily transportable to carry out in-situ measures. 
To avoid some of the drawbacks associated with usual techniques, we propose a new experimental method to determine the liquid/gas equilibrium characteristics of a volatile compound. This includes the saturating vapor pressure, the sorption isotherm and the HLC. Since it is involved in many industrial soil contaminations, the particular case of trichloroethylene (TCE) will be investigated.

One of today's concerns for the environment relates to remediation technologies of contaminant absorbed in natural soils and the influence of environmental conditions $[1,23]$. In such porous material, the water phase is adsorbed onto the solid phase surface and its physical properties are modified by liquid/solid interactions. These microscale effects account for the hygroscopic nature of soil and become predominant when clays or organic matters are present $[20,24]$. One main issue addressed in this paper is whether the thermodynamic equilibrium between the components of a solution (water and solute) and their vapor are modified in a hygroscopic soil when compared to the same aqueous solution out of the soil. Indeed, previous experimental results underlined that the hydric state has a significant role on vapor sorption of volatile organic compound and can reduce the sorption capacities about 2 orders of magnitude $[1,22]$. However, vapor sorption of volatile organic compound in unsaturated soil has not been investigated based on Henry's law. If competitive sorption behavior can occur at very low water content [22], once the solid interface available for vapor sorption becomes negligible, the vapor dissolves in adsorbed water films.

\section{Materials and Methods}

2.1. Theoretical Background. The main equilibrium characteristic that describes the adsorption of a volatile compound in a porous medium is the sorption isotherm curve. It is represented by the plot of the liquid mass content, $w_{l}$, as a function of the liquid activity, $a_{l}$. At equilibrium, the liquid activity, $a_{l}$, can be determined through the partial pressure of the vapor, $P_{\text {veq }}$, surrounding the porous medium by

$$
a_{l}=\frac{P_{\mathrm{veq}}}{P_{\mathrm{vsat}}},
$$

where $P_{\text {vsat }}$ is the saturating vapor pressure.

In this situation, the liquid activity characterizes the bonding energy between the liquid phase and the surface of the porous microstructure. This manifests itself at the macroscopic scale by the hygroscopic nature of the porous medium. The sorption isotherm curve defines a liquid content range, generally called the hygroscopic domain, where the equilibrium vapor pressure of the liquid phase is below its saturating value measured outside of the porous medium. The most common methods are only dedicated to the characterization of water sorption and are widely used in agronomy, food engineering or drying technology. Since the water activity is also equal to the equilibrium relative humidity, $\mathrm{RH}$, water sorption behavior is generally established using standard saturated salt solutions. Characterization of sorption isotherm in the case of any other volatile compound requires more sophisticated experimental device.
When this volatile compound is dissolved in an aqueous solution, Henry's law establishes a sharing relation between the aqueous phase and the gas phase. This law is generally described by a linear function and leads to express as a dimensional constant, $K_{H}\left[\mathrm{~Pa} \cdot \mathrm{m}^{3} \cdot \mathrm{mol}^{-1}\right]$ :

$$
K_{H}=\frac{P_{\mathrm{Teq}}}{C_{T}},
$$

where $P_{\mathrm{Teq}}$ is the vapor partial pressure of the volatile compound in the surrounding atmosphere at equilibrium and $C_{T}$ is its concentration in the aqueous solution.

The case of an aqueous solution adsorbed in a porous medium has not been yet clearly investigated in a unique description. The hygroscopic nature of porous materials should obviously interfere with Henry's law since the thermodynamic state of the liquid phase is modified by adsorption phenomena. This issue will be addressed in the last part of this work.

2.2. Preparation of Samples. Solution of TCE is prepared with distilled water at a given initial concentration $C_{T}^{0}$ and stored hermetically at $30^{\circ} \mathrm{C}$ for 10 minutes to enable the solution to homogenize. A desired volume $V_{l}$ of the solution is extracted using a microsyringe and injected directly in the sample holder.

In case of soil sample, the liquid phase or solution under investigation is mixed with a natural soil, silty sand with a $10 \%$ clayey fraction [29]. After being oven dried for $24 \mathrm{~h}$ at $105^{\circ} \mathrm{C}$, the required amount of soil, $m_{s}=18.1 \mathrm{~g}$ and liquid compound are mixed using a high precision scale $\left(10^{-4} \mathrm{~g}\right)$. Homogeneity of liquid content is ensured by storing the mixture in an airtight container for $24 \mathrm{~h}$. Then, the soil mixture is compacted in a cylindrical ring $(32 \mathrm{~mm}$ diameter and $15 \mathrm{~mm}$ high) to reach a dry density of $\rho_{s}=1500 \mathrm{~kg} \cdot \mathrm{m}^{-3}$, which corresponds to a porosity of $\phi=43 \%$. Many samples are prepared at different liquid contents to cover the full range of liquid activity.

2.3. Measurement Method of the Equilibrium Vapor Pressure of a Pure Liquid. The experimental device has been initially developed to determine the water activity and has thus been called activity meter. The principle of measure is briefly synthesized below since the extension proposed in next section will be based upon this initial development. Further details about the method can be found in a previous publication [30].

The experimental device is sketched in Figure 1 while main dimensions are synthesized in Table 1 . The liquid phase or sample to be characterized (a) is disposed inside a sealed chamber whose volume is controlled by a piston (b) and a micrometric screw gauge (c), so as to impose negative relative gas pressure. In addition, the total pressure of the gas phase, $P_{g}$, and its temperature, $T$, are recorded along the test through a temperature thermocouple (d) and a pressure transducer (Druck, PMP4030AB) (e). Isothermal conditions are ensured by immersing the whole device in a thermoregulated bath.

The gas phase is considered thermodynamically closed and is composed of dry air and vapor of the volatile compound under investigation. At equilibrium, the vapor partial 


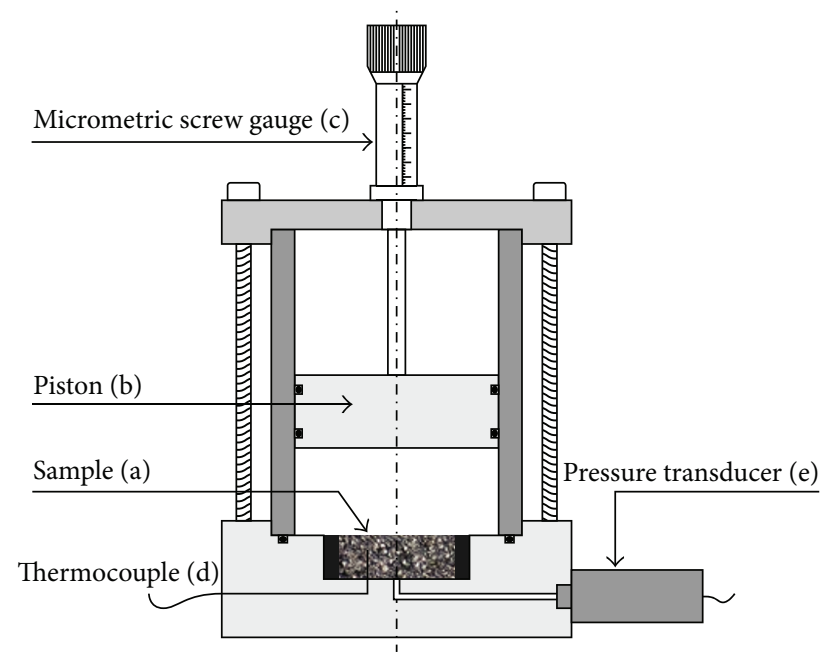

FIGURE 1: Schema of the activity meter.

TABLE 1: Main dimensions of the experimental device (Figure 1).

\begin{tabular}{lc}
\hline Sample diameter & $32 \mathrm{~mm}$ \\
Sample height & $15 \mathrm{~mm}$ \\
Sample volume & $12.07 \mathrm{~cm}^{3}$ \\
Piston diameter & $74 \mathrm{~mm}$ \\
Maximum displacement of piston & $100 \mathrm{~mm}$ \\
\hline
\end{tabular}

pressure is equal to its equilibrium value, $P_{\text {veq }}$, and the total gas pressure, $P_{g}$, can be expressed by

$$
P_{g}=P_{\text {veq }}+P_{a}=P_{\text {veq }}+n_{a} \frac{R T}{V_{g}}=P_{\text {veq }}+n_{a} \frac{R T}{V_{0}+V},
$$

where the dry air pressure, $P_{a}$, is assumed to behave as an ideal gas that depends on its mole number inside the chamber, $n_{a}$, the ideal gas constant, $R$, the temperature, $T$ and the volume of the gas phase, $V_{g}$. The gas phase volume can be decomposed as the sum of an initial volume, $V_{0}$, and an additional volume, $V$, imposed by the displacement of the piston. In (3), the values of $P_{\text {veq }}, n_{a}$, and $V_{0}$ are unknown while the gas phase mixture is continuously characterized by $P_{g}, T$, and $V$. We can fairly consider the equilibrium vapor pressure, $P_{\text {veq }}$, as a constant since the amount of water evaporated during one experiment is negligible and the liquid content remains the same. Therefore, 3 equilibrium situations with different values of $P_{g}, T$, and $V$ are required to determine the 3 unknowns $P_{\text {veq }}, n_{a}$, and $V_{0}$.

On this basis, the principle of an experiment consists in creating various equilibrium situations by moving up the piston and providing set of value triplets $\left(P_{g}^{i}, T^{i}, V^{i}\right)$ to be introduced in (3) where the superscript $i$ relates to the successive equilibrium situations. While 3 equilibrium stages are mathematically sufficient to identify the 3 unknowns, the measure accuracy if highly increased by relying on 6 to $10 \mathrm{vol}-$ ume increments. The resulting set of equations is solved using a nonlinear least-squares minimization numerical procedure.

This method has already been validated in the case of water using 2 approaches [30]. In the first one, the water vapor pressure regulated by a standard saturated salt solution was measured and compared to the water activity of such solution specified by the French National Standards Association (NF $X$ 15119). Errors less than $1 \%$ were obtained with 5 different saturated salt solutions covering the whole range of water activities. In the second approach, a comparison with another technique for determining the water sorption isotherm of a reference soil was carried out [29]. Both methods led to almost identical sorption isotherm curves asserting the accuracy of the technique proposed in this work.

2.4. Extension of the Method to a Mixture. The objective is to extend the mechanical method to measure the vapor partial pressures above a mixture of water and TCE. Compared to the procedure described previously (3), the vapor partial pressure of TCE, $P_{\text {Teq }}$, represents an additional unknown. Considering that the gas phase contained in the device behaves as an ideal gas mixture, every equilibrium state is characterized by the following equation:

$$
P_{g}=P_{\mathrm{veq}}+P_{\mathrm{Teq}}+P_{a}=P_{\mathrm{veq}}+P_{\mathrm{Teq}}+n_{a} \frac{R T}{V_{0}+V}
$$

If the equilibrium vapor pressure of water, $P_{\text {veq }}$, can be assumed to be constant throughout the experiment, the equilibrium vapor pressure of TCE, $P_{\text {Teq }}$, varies substantially because it is highly volatile and the amount of solution is small. Contrary to previous situation, the multiplication of volume increments cannot allow to determine all the unknowns. An alternative is to rely on the high volatility of TCE. Indeed, the total mass balance equation of TCE in liquid and gas phases can be written in terms of mole number:

$$
C_{T} V_{l}+\frac{P_{\text {Teq }} V_{g}}{R T}=C_{T}^{0} V_{l}=C^{t e},
$$

where $C_{T}^{0}$ is the initial concentration of TCE, $V_{l}$ the volume of the aqueous phase and $C_{T}$ the actual concentration at the corresponding increment. If almost all the TCE has evaporated from the $i$ th increment corresponding to a threshold value of the gas volume, $V_{g} \geqslant V_{\text {thresh }}$, its equilibrium vapor pressure, $P_{\text {Teq }}$, becomes very low and drops below the accuracy of the pressure sensor. Considering $V_{l}=1 \mathrm{~cm}^{3}$ of solution (water + TCE) at saturation, evolution of the equilibrium vapor pressure of TCE, $P_{\text {Teq }}$, as a function of gas phase volume imposed by the piston, $V_{g}$, is calculated using (5) and plotted in Figure 2.

It evidences that for $V_{g} \geqslant V_{\text {thresh }}=300 \mathrm{~cm}^{3}$, the TCE vapor pressure is below $10 \mathrm{~Pa}$ and can reasonably be neglected in the expression of the total equilibrium gas pressure (4). It becomes

$$
P_{g}=P_{\text {veq }}+n_{a} \frac{R T}{V_{0}+V}
$$

and corresponds to the case analyzed in previous section (3). 


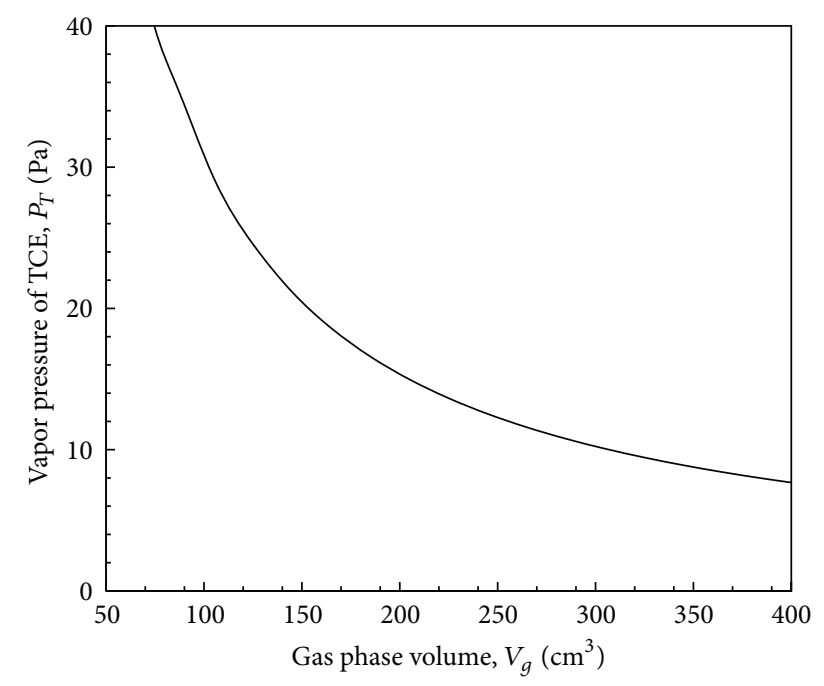

FIGURE 2: Equilibrium vapor pressure of TCE as function of piston volume.

Thus, by taking into account this point, the method relies on 2 stages:

(1) A slight volume increment is imposed to obtain a vapor equilibrium at ambient pressure. This equilibrium is described by (4) providing the 1st triplet $\left(P_{g}^{1}\right.$, $\left.T^{1}, V^{1}\right)$.

(2) A large volume increment is imposed in order to evaporate all the dissolved TCE and make its vapor pressure being negligible. From this point, vapor equilibrium is described by (6) and an identical procedure as detailed in previous section can be applied; that is, successive volume increments lead to a set of equilibrium situations characterized by value triplets $\left(P_{g}^{i}, T^{i}, V^{i}\right)$ with $2 \leq i \leq n$.

The last triplets $(2 \leq i \leq n)$ are introduced in (6) and solved using a nonlinear least-squares minimization numerical procedure to determine the 3 unknowns $\left(P_{\text {veq }}, n_{a}\right.$, $\left.V_{0}\right)$. Then, (4) allows calculating the TCE vapor pressure, $P_{\text {Teq, }}$, in the first equilibrium stage and (5) leads to determining the corresponding aqueous concentration of TCE, $C_{T}$. Finally, the pair of value $\left(C_{T}, P_{\text {Teq }}\right)$ can be used to evaluate the HLC based on (2).

Therefore, the experimental procedure to be applied must comply with the 2 constraints: the piston volume $V_{g} \geqslant$ $V_{\text {thresh }}=300 \mathrm{~cm}^{3}$ (see Table 1) and the volume of solution injected in the device $V_{l} \leqslant 1 \mathrm{~cm}^{3}$. As these constraints have been set up with a solution at saturation, results would even be more accurate for a lower concentration. All the tests are performed at $30^{\circ} \mathrm{C}$ by immersing the experimental device in a thermoregulated bath. About 30 minutes are required to achieve thermal and liquid/gas equilibrium before starting the test. The experimental procedure is similar to the one described in previous section; once the whole device is at thermodynamic equilibrium, volume increments are made using the screw system to create imbalances and total gas
TABLE 2: Saturating vapor pressures of TCE at different temperatures [25].

\begin{tabular}{lcc}
\hline & $\begin{array}{c}\text { This work } \\
{[\mathrm{Pa}]}\end{array}$ & $\begin{array}{c}\text { Literature } \\
{[\mathrm{Pa}]}\end{array}$ \\
\hline TCE at $293 \mathrm{~K}$ & 7759 & 7601 \\
TCE at $303 \mathrm{~K}$ & 11900 & 12220 \\
\hline
\end{tabular}

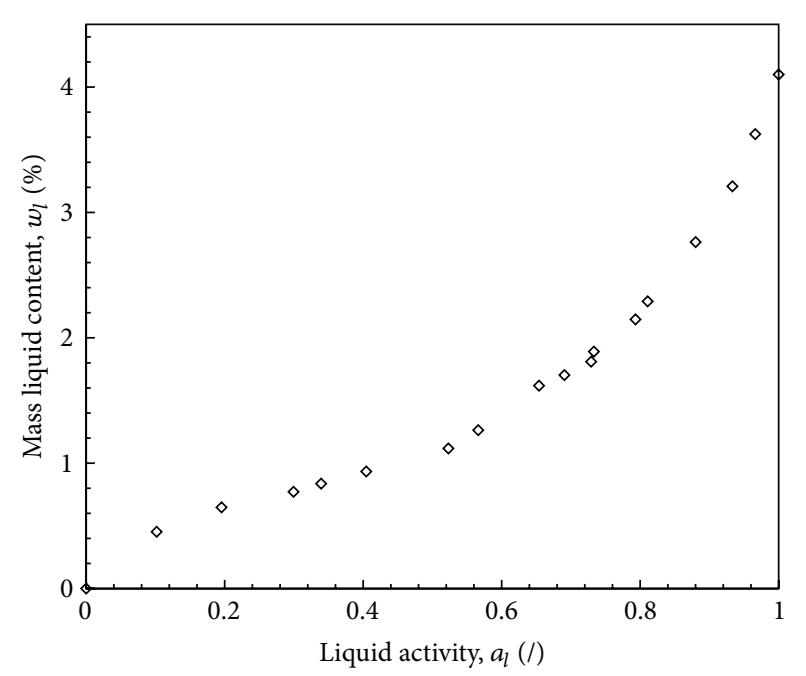

FIGURE 3: Sorption isotherm curves of TCE adsorbed in a clayey silty sand determined with the activity meter.

pressure and temperature are continuously recorded until a new equilibrium state is reached.

\section{Results and Discussion}

3.1. Case of a Pure Liquid Phase. The main advantage of this method is that it can be used with any volatile compound. The case of TCE is presented here since this volatile organic compound is involved in many industrial soil contaminations. A large range of saturating vapor pressure is available in the literature, varying from 7600 to $8600 \mathrm{~Pa}$ in case of TCE at $20^{\circ} \mathrm{C}$. Thus, the measures obtained with this method are in agreement with reference values from the literature (Table 2).

3.2. Case of a Pure Liquid Phase Absorbed in a Natural Soil. Identical experimental procedure is used to characterize the sorption isotherm of pure TCE adsorbed in a natural soil. The plot of liquid mass content, $w_{l}$, as a function of liquid activity, $a_{l}$ is shown in Figure 3. Data from literature indicate that the upper limit of the hygroscopic domain is difficult to characterize $[31,32]$ and requires many days to reach equilibrium. As this method directly measures the activity of the liquid instead of imposing it based on a regulated atmosphere, the time required for measurement is about 4 hours.

Apart from the usual sorption isotherm of water, this method allows us to determine the sorption isotherm of any compound as long as its equilibrium vapor pressure is large enough to be measured by using a pressure sensor. This clearly shows that hygroscopic effects dominate at 


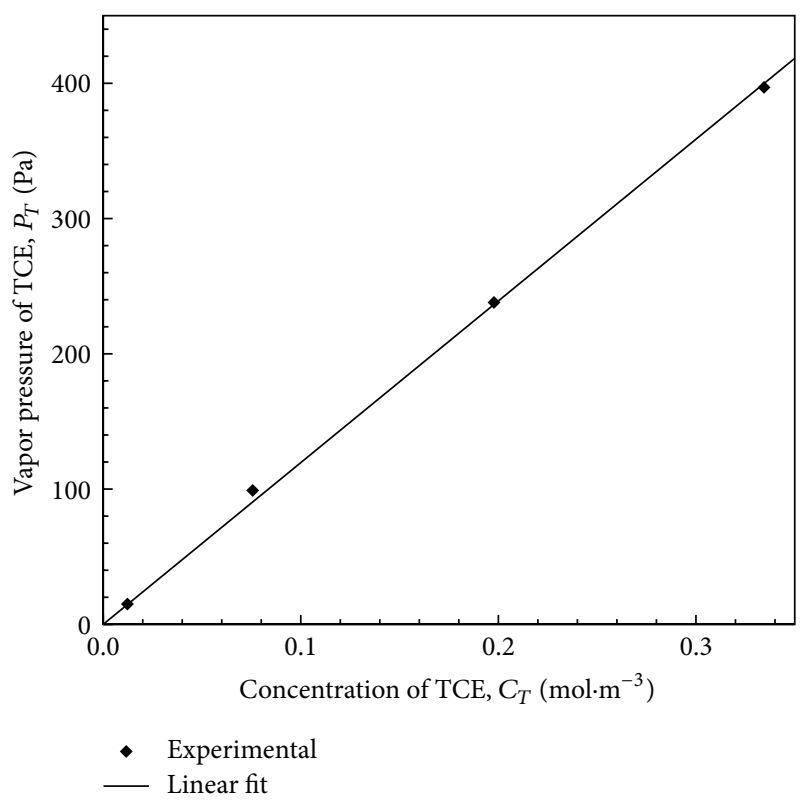

FIGURE 4: Vapor partial pressure of TCE as a function of its aqueous concentration.

TABLE 3: Values of Henry's law constant $\left[\mathrm{Pa} \cdot \mathrm{m}^{3} \cdot \mathrm{mol}^{-1}\right]$ of TCE at $303 \mathrm{~K}$.

\begin{tabular}{lc}
\hline Authors & $K_{H}\left[\mathrm{~Pa} \cdot \mathrm{m}^{3} \cdot \mathrm{mol}^{-1}\right]$ \\
\hline Gossett [26] & 1237 \\
Ashworth et al. [9] & 1297 \\
Tse et al. [27] & 1155 \\
Robbins et al. [7] & 1327 \\
Heron et al. [3] & 1158 \\
Staudinger and Roberts [28] & 1260 \\
This work & 1195 \\
\hline
\end{tabular}

low liquid content, not only for only water, but also with TCE. This sorption isotherm was obtained at zero relative humidity conditions. Indeed, several works have underlined the influence of moisture on vapor sorption of TCE into soils and this issue will be investigated in the next section.

3.3. Henry's Law Constant of a Solution of TCE. Some experiments have been carried out to determine the HLC of TCE based on the previously described method. Using 4 different solutions of TCE $\left(V_{l}=1 \mathrm{~cm}^{3}\right.$ prepared at various $\left.C_{T}^{0}\right)$, pairs of value $\left(C_{T}, P_{\text {Teq }}\right)$ obtained are plotted in Figure 4. The relation between the vapor pressure of TCE and its aqueous concentration is fairly described by a linear function that verifies Henry's law. The HLC evaluated in this work is compared with those from the literature in Table 3 showing a good agreement with previous results.

3.4. Henry's Law Constant of a Solution of TCE Absorbed in a Natural Soil. As presented in previous section, adsorption phenomena in a natural soil modify the thermodynamic state of the liquid phase. These deviations, also called hygroscopic

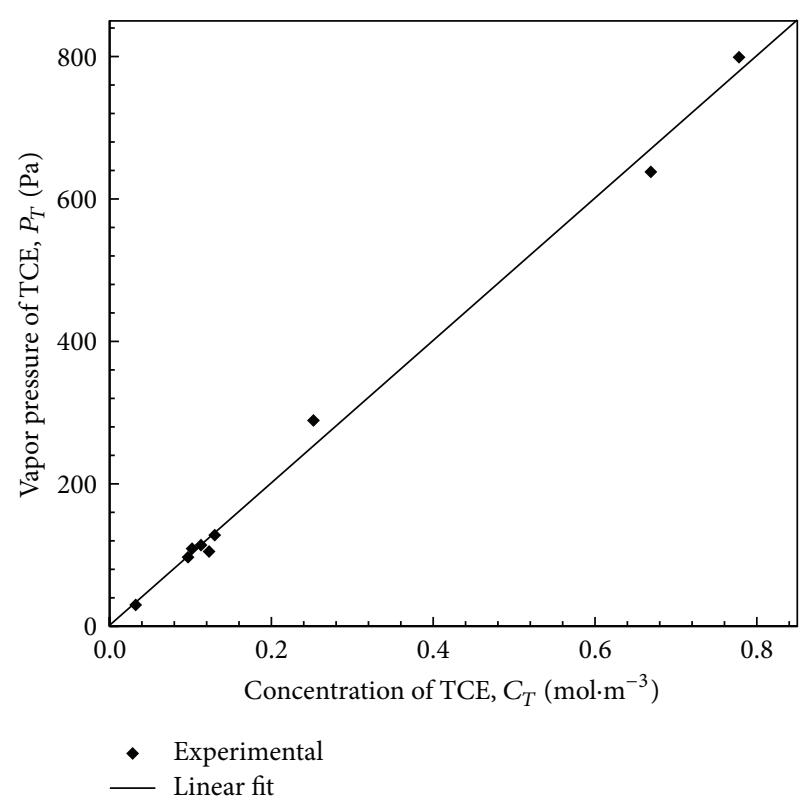

FIGURE 5: Vapor partial pressure of TCE as a function of its aqueous concentration in case of a solution adsorbed in a natural soil.

effects, are characterized by the liquid activity and are generally described through the sorption isotherm curve (Figure 3). At this point, the objectives are to investigate the validity of Henry's law when hygroscopic effects are significant and how the HLC is affected. The natural soil is the same than the one used in previous section. Tests involve a wide range of initial concentration, $C_{T}^{0}$, from 0.84 to $7.6 \mathrm{~mol} \cdot \mathrm{m}^{-3}$ and various mass liquid contents, $w_{l}$, from $1 \%$ to $7 \%$.

The representation of vapor pressure of TCE as function of its concentration in the liquid phase is given in Figure 5. It shows that the experimental points are almost aligned and can be well represented by a linear relation. This linear dependence can be assimilated to Henry's law validating its formulation in case of solution adsorbed in a soil. No significant influence of the liquid content has been highlighted meaning that a single HLC can be determined. Following its definition given in (2), the HLC in soil estimated in this work is

$$
K_{H}^{s}=1002 \mathrm{~Pa} \cdot \mathrm{m}^{3} \cdot \mathrm{mol}^{-1} .
$$

This value is lower than the one determined outside of the soil (Table 3 ) of about $16 \%$. Indeed, for a given concentration of TCE, the vapor partial pressure will be lower when the solution of TCE is adsorbed in soil. This quantitative difference is probably due to the liquid-solid binding energy associated with adsorption phenomena, which becomes significant at low liquid content when hygroscopic effects are predominant especially in the presence of a significant clayey fraction. Further studies are required using various soil compositions to carefully check this issue.

3.5. Desorption Isotherm of Water in the Case of a Mixture of Water and TCE. Through the experimental procedure described above, the vapor partial pressure of water, $P_{\text {veq }}$, has 


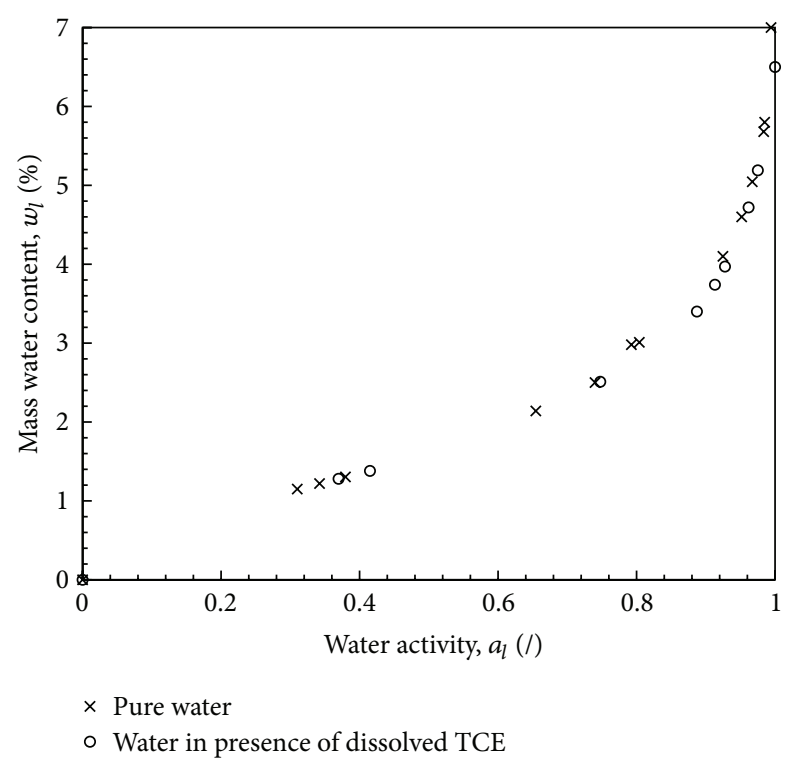

FIGURE 6: Comparison of water sorption isotherm curves determined with and without the presence of dissolved TCE.

also been determined. This therefore allows us to compare the equilibrium vapor pressure of water measured with pure water adsorbed in soil [30] to the one obtained in the case of a mixture of water and TCE. The comparison of both curves presented in Figure 6 does not highlight any noticeable deviation associated with the presence of TCE. Even if TCE does not significantly modify the equilibrium vapor pressure of water, several authors have shown that the water content plays an important role in the mechanism of sorption and desorption of organic compounds [1].

3.6. Discussion. The experimental device originally designed to determine the water activity in porous media [30] has been extended to the analysis of sorption characteristics of TCE as a pure liquid phase or dissolved in aqueous solution. For the sake of validation, the method and the experimental device developed have been applied to evaluate the saturating vapor pressure and the HLC of TCE at $30^{\circ} \mathrm{C}$. Experimental values obtained are in good agreement with the literature. The major benefits are that this approach is generally much faster and less expensive than previously proposed methods. When compared to existing devices, this method is easy to carry out and necessitates only classical and costless measurement sensors, a type- $\mathrm{K}$ thermocouple and a gas pressure transducer. The volume variations can be accurately imposed with a micrometric screw system allowing automation of the process. No chemical product or delicate sensor is required and the whole device is easily transportable allowing in situ measurements.

The method can easily be extended to measure the sorption characteristics of a VOC adsorbed in soil. It has been shown that partition between dissolved TCE and gaseous TCE is also governed by a linear relation similar to Henry's law. With the clayey silty sand used in this work, a decrease of HLC of about $16 \%$ is observed. This variation can be attributed to hygroscopic effects that represent the fixing of TCE onto the clayey fraction of the soil. No dependence on the liquid content has been evidenced in this case and a single HLC gives an accurate description of the sorption of TCE over wide ranges of TCE concentration and liquid content. Furthermore, the water-vapor equilibrium is not affected by the presence of TCE since identical water sorption isotherm curves are obtained with or without TCE. Even if the influence of moisture on the sorption behavior of TCE in soils have already been highlighted $[1,20,22,24]$, it was not investigated in the framework of Henry's law.

These results, restricted in this work to the study of TCE adsorbed in a particular soil, must be confirmed with various soils having different texture and composition. The experimental device presented in this paper and the associated procedure should facilitate the extension of such experimental data.

\section{Conflict of Interests}

The authors declare that there is no conflict of interests regarding the publication of this paper.

\section{References}

[1] T.-F. Lin, J. C. Little, and W. W. Nazaroff, "Transport and sorption of volatile organic compounds and water vapor within dry soil grains," Environmental Science \& Technology, vol. 28, no. 2, pp. 322-330, 1994.

[2] J. Staudinger and P. V. Roberts, "A critical review of henry's law constants for environmental applications," Critical Reviews in Environmental Science and Technology, vol. 26, no. 3, pp. 205297, 1996.

[3] G. Heron, T. H. Christensen, and C. G. Enfield, "Henry's law constant for trichloroethylene between 10 and 95०C," Environmental Science and Technology, vol. 32, no. 10, pp. 1433-1437, 1998.

[4] J. Altschuh, R. Brüggemann, H. Santl, G. Eichinger, and O. G. Piringer, "Henry's law constants for a diverse set of organic chemicals: experimental determination and comparison of estimation methods," Chemosphere, vol. 39, no. 11, pp. 1871-1887, 1999.

[5] J. B. Falabella, A. C. Kizzie, and A. S. Teja, "Henry's constants of gases and volatile organic compounds in aqueous solutions," Fluid Phase Equilibria, vol. 241, no. 1-2, pp. 96-102, 2006.

[6] F. Gharagheizi, A. Eslamimanesh, A. H. Mohammadi, and D. Richon, "Empirical method for estimation of Henry's law constant of non-electrolyte organic compounds in water," Journal of Chemical Thermodynamics, vol. 47, pp. 295-299, 2012.

[7] G. A. Robbins, S. Wang, and J. D. Stuart, "Using the static headspace method to determine Henry's law constants," Analytical Chemistry, vol. 65, no. 21, pp. 3113-3118, 1993.

[8] C. Ji and E. M. Evans, "Using an internal standard method to determine Henry's law constants," Environmental Toxicology and Chemistry, vol. 26, no. 2, pp. 231-236, 2007.

[9] R. A. Ashworth, G. B. Howe, M. E. Mullins, and T. N. Rogers, "Air-water partitioning coefficients of organics in dilute aqueous solutions," Journal of Hazardous Materials, vol. 18, no. 1, pp. 25-36, 1988. 
[10] T. Shimotori and W. A. Arnold, "Measurement and estimation of Henry's law constants of chlorinated ethylenes in aqueous surfactant solutions," Journal of Chemical and Engineering Data, vol. 48, no. 2, pp. 253-261, 2003.

[11] X.-S. Chai, J. B. Falabella, and A. S. Teja, "A relative headspace method for Henry's constants of volatile organic compounds," Fluid Phase Equilibria, vol. 231, no. 2, pp. 239-245, 2005.

[12] J. Dewulf, H. van Langenhove, and P. Everaert, "Determination of Henry's law coefficients by combination of the equilibrium partitioning in closed systems and solid-phase microextraction techniques," Journal of Chromatography A, vol. 830, no. 2, pp. 353-363, 1999.

[13] J. M. Roberts, "Measurement of the Henry's law coefficient and first order loss rate of pan in n-octanol," Geophysical Research Letters, vol. 32, Article ID L08803, pp. 1-4, 2005.

[14] H. S. S. Ip, X. H. H. Huang, and J. Z. Yu, "Effective Henry's law constants of glyoxal, glyoxylic acid, and glycolic acid," Geophysical Research Letters, vol. 36, no. 1, Article ID L01802, 2009.

[15] S. H. Lee, S. Mukherjee, B. Brewer, R. Ryan, H. Yu, and M. Gangoda, "A laboratory experiment to measure Henry's law constants of volatile organic compounds with a bubble column and a gas chromatography flame ionization detector (GC-FID)," Journal of Chemical Education, vol. 90, no. 4, pp. 495-499, 2013.

[16] J. D. Kish, C. Leng, J. Kelley, J. Hiltner, Y. Zhang, and Y. Liu, "An improved approach for measuring Henry's law coefficients of atmospheric organics," Atmospheric Environment, vol. 79, pp. 561-565, 2013.

[17] S. A. Brockbank, J. L. Russon, N. F. Giles, R. L. Rowley, and W. V. Wilding, "Infinite dilution activity coefficients and Henry's law constants of compounds in water using the inert gas stripping method," Fluid Phase Equilibria, vol. 348, pp. 45-51, 2013.

[18] K. N. McPhedran, R. Seth, and K. G. Drouillard, "Evaluation of the gas stripping technique for calculation of Henry's law constants using the initial slope method for 1,2,4,5tetrachlorobenzene, pentachlorobenzene, and hexachlorobenzene," Chemosphere, vol. 91, no. 11, pp. 1648-1652, 2013.

[19] D. Richon, "New equipment and new technique for measuring activity coefficients and henry's constants at infinite dilution," Review of Scientific Instruments, vol. 82, no. 2, Article ID 025108, 2011.

[20] S. M. Steinberg, J. S. Schmeltzer, and D. K. Kreamer, "Sorption of benzene and trichloroethylene (TCE) on a desert soil: effects of moisture and organic matter," Chemosphere, vol. 33, no. 5, pp. 961-980, 1996.

[21] L. H. Turner, Y. C. Chiew, R. C. Ahlert, and D. S. Kosson, "Measuring vaporliquid equilibrium for aqueous-organic systems: review and a new technique," AIChE Journal, vol. 42, no. 6, pp. 1772-1788, 1996.

[22] D. R. Unger, T. T. Lam, C. E. Schaefer, and D. S. Kosson, "Predicting the effect of moisture on vapor-phase sorption of volatile organic compounds to soils," Environmental Science \& Technology, vol. 30, no. 4, pp. 1081-1091, 1996.

[23] D. I. Oh, K. Nam, J. W. Park, J. H. Khim, Y. K. Kim, and J. Y. Kim, "Impacts of environmental conditions on the sorption of volatile organic compounds onto tire powder," Journal of Hazardous Materials, vol. 153, no. 1-2, pp. 157-163, 2008.

[24] C. T. Chiou and T. D. Shoup, "Soil sorption of organic vapors and effects of humidity on sorptive mechanism and capacity," Environmental Science \& Technology, vol. 19, no. 12, pp. 11961200, 1985.
[25] A. Seidel, Ed., Kirk-Othmer Encyclopedia of Chemical Technology, Wiley-Interscience, John Wiley \& Sons, New York, NY, USA, 5th edition, 2007.

[26] J. M. Gossett, "Measurement of Henry's law constants for $C_{1}$ and $\mathrm{C}_{2}$ chlorinated hydrocarbons," Environmental Science \& Technology, vol. 21, no. 2, pp. 202-208, 1987.

[27] G. Tse, H. Orbey, and S. I. Sandler, "Infinite dilution activity coefficients and Henry's law coefficients of some priority water pollutants determined by a relative gas chromatographic method," Environmental Science \& Technology, vol. 26, no. 10, pp. 2017-2022, 1992.

[28] J. Staudinger and P. V. Roberts, “A critical compilation of Henry's law constant temperature dependence relations for organic compounds in dilute aqueous solutions," Chemosphere, vol. 44, no. 4, pp. 561-576, 2001.

[29] A.-L. Lozano, F. Cherblanc, B. Cousin, and J.-C. Bénet, "Experimental study and modelling of the water phase change kinetics in soils," European Journal of Soil Science, vol. 59, no. 5, pp. 939949, 2008.

[30] S. Ouoba, F. Cherblanc, B. Cousin, and J.-C. Bénet, "A new experimental method to determine the sorption isotherm of a liquid in a porous medium," Environmental Science \& Technology, vol. 44, no. 15, pp. 5914-5919, 2010.

[31] P. Baucour and J. D. Daudin, "Development of a new method for fast measurement of water sorption isotherms in the high humidity range Validation on gelatine gel," Journal of Food Engineering, vol. 44, no. 2, pp. 97-107, 2000.

[32] T. Rougier, C. Bonazzi, and J.-D. Daudin, "Modeling incidence of lipid and sodium chloride contents on sorption curves of gelatin in the high humidity range," LWT-Food Science and Technology, vol. 40, no. 10, pp. 1798-1807, 2007. 

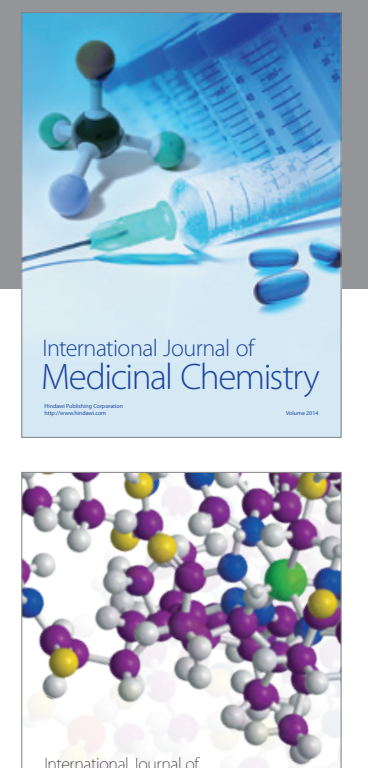

\section{Carbohydrate} Chemistry

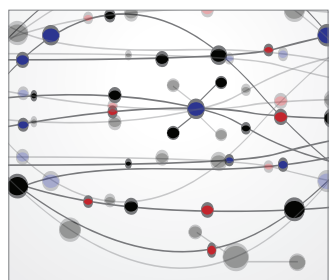

The Scientific World Journal
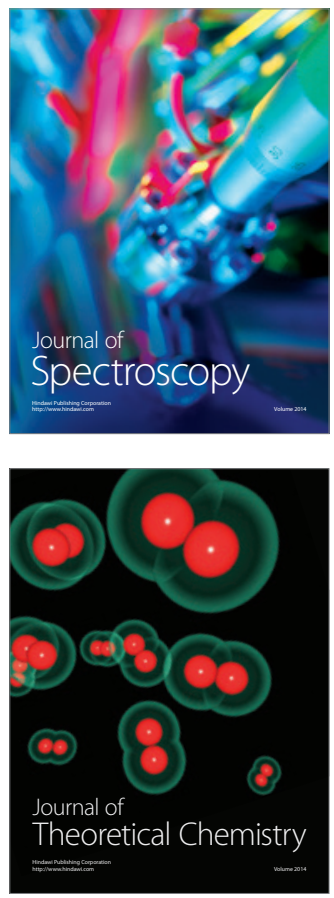
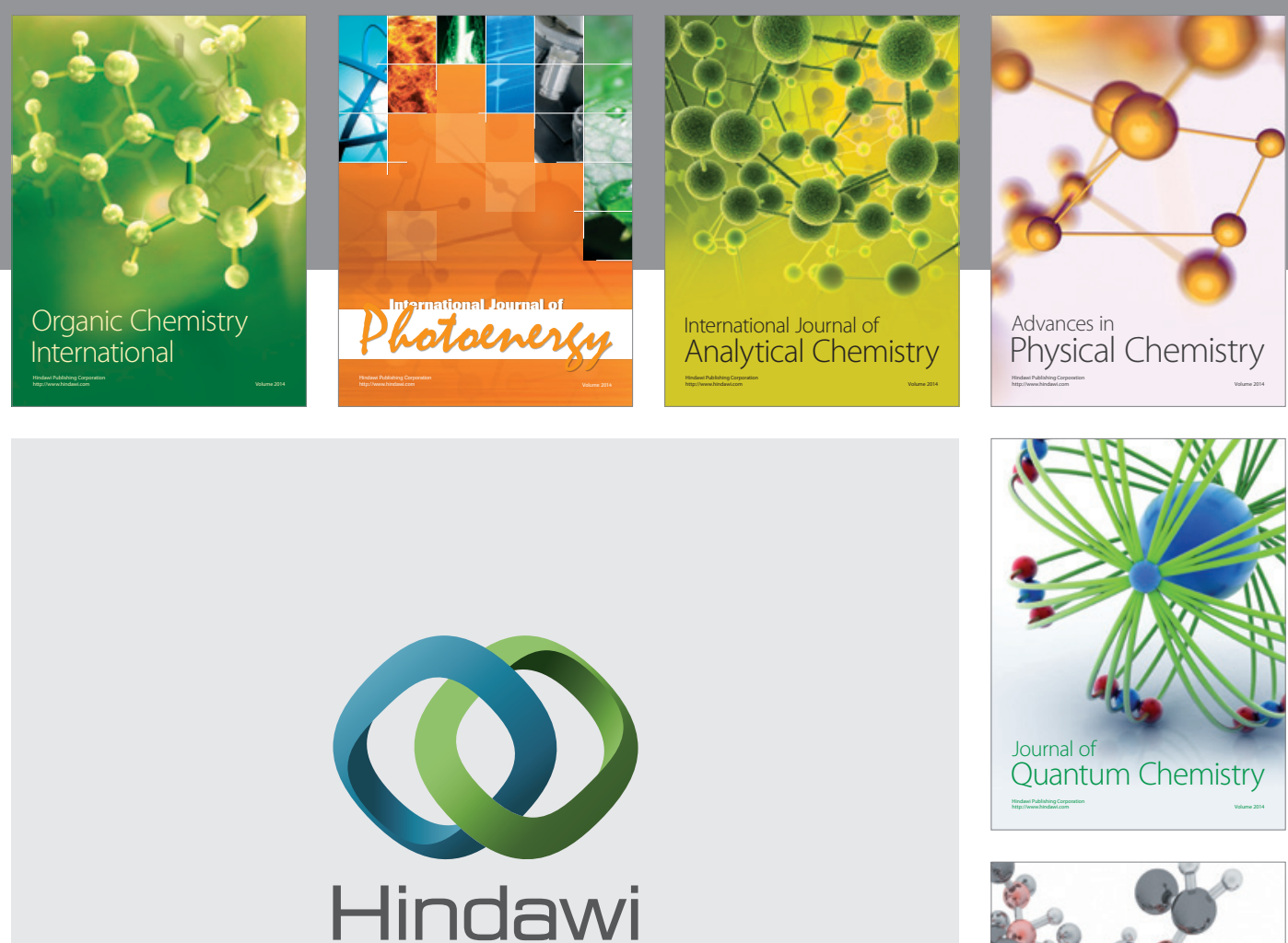

Submit your manuscripts at

http://www.hindawi.com

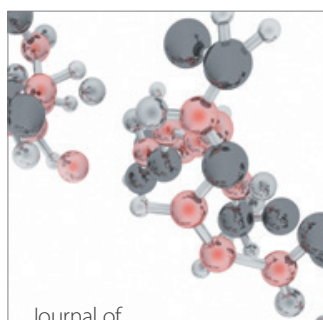

Analytical Methods

in Chemistry

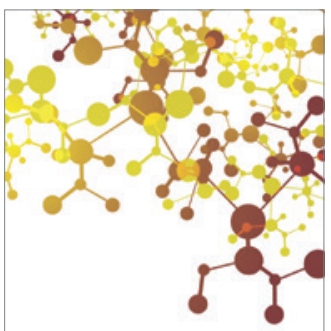

Journal of

Applied Chemistry

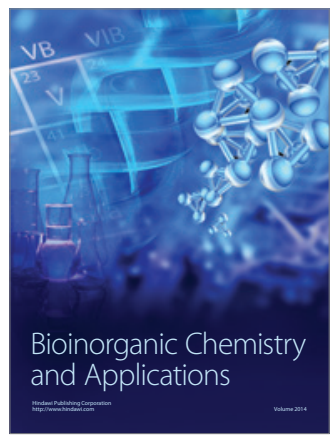

Inorganic Chemistry
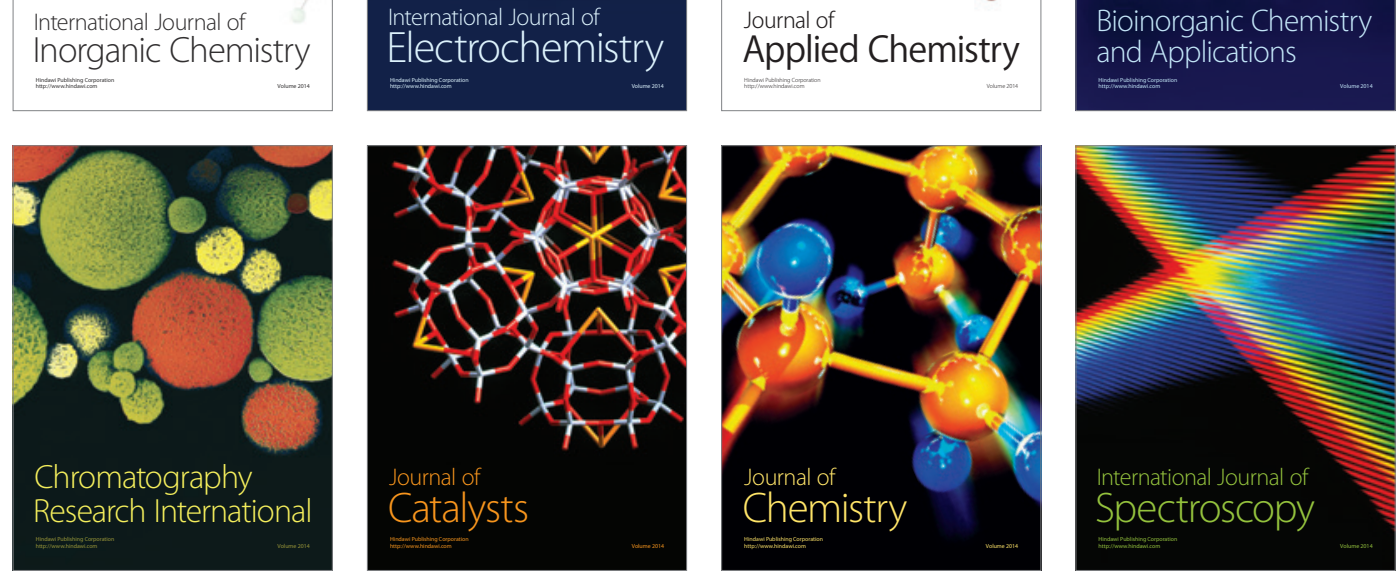